\title{
Measuring the Eccentricity of the Terrestrial Orbit: An Experiment in the Classroom
}

\author{
By R. Szostak \\ Universität Münster, Institut für Didaktik der Physik, Germany
}

\section{The eccentricity is very small}

Most textbooks of physics present the terrestrial orbit by a drawing which shows an ellipse of substantial eccentricity. This suggests a remarkable variation of the distance between Sun and Earth during the year up to a value of about 3:1 and more. Imagine the dramatic variation in size of the radiating area of the Sun seen by the terrestrial inhabitants with all the terrible consequences for temperature. All this is not true. There is no obvious change in size of the solar disc.

In nature the numerical eccentricity of the terrestrial orbit is only $\epsilon=e / a=0.01675$ ( $a=$ major or long axis, $b=$ minor or short axis, $e=$ focal length). This value is so small, that this ellipse cannot be distinguished from a circular orbit in a drawing when using a normal pen $(a / b=1.00014)$. The deviation would be $1 / 20$ of the width across the line of the pencil. By what procedure would it be possible to measure this small eccentricity using only simple means in the classroom?

\section{Observe the varying size of the solar disc}

A first approach could be the idea to take photographs of the Sun throughout the whole year. The angular width of the solar disc varies by about $3 \%$ within this period. The focal length $f=50 \mathrm{~mm}$ of a normal camera produces an image of the Sun, which is $0.4 \mathrm{~mm}$ in diameter on the film. Trying to determine the eccentricity from these pictures better than $10 \%$ would mean ability to measure difference of $1 \mu \mathrm{m}$ in size on the film. So this procedure will not work well. It will be necessary to use a camera with a considerably larger focal length or a telescope with a magnifying factor which has to be kept reliably constant throughout the year.

But we can be independent of this condition by measuring the angular width of the solar disc via the daily rotation of the earth. In this case it is sufficient to measure the motion of the solar image on a screen, produced by a telescope at rest. The Sun exhibits its angular size by the time elapsed in passing a line on the screen. This happens within about 2 minutes. For determining the variations of the angular width to better than $10 \%$, it is necessary to measure the time of this passage to within $0.3 \mathrm{~s}$. This must be done carefully. In addition there is the problem that the brightness of the solar disc drops near its contouring edge. So we have some little problems to determine the eccentricity of the terrestrial orbit.

\section{Variation in solar time}

But there is another way to determine it; this method gives results with a few percent error which can be obtained with almost no technical equipment: there is a phenomenon which was well known already the Greeks in ancient times: the seasons, whose beginning and ending are given by the dates of the equinoxes and solstices, are not equally long. They differ by up to 5 days, as one may find in any calendar. In ancient times people 


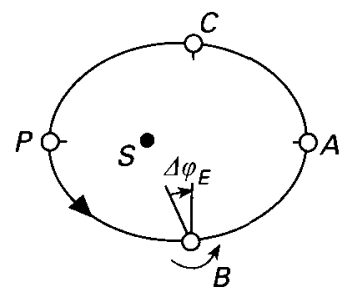

FIGURE 1.

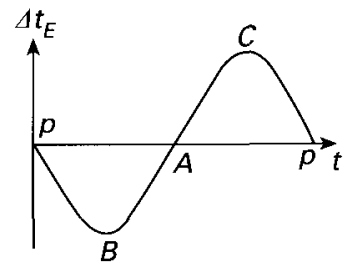

FIGURE 2.

thought that the Sun was moving around the Earth, which was at rest. So these deviations were understood as an eccentric motion of the Sun. Changing to the Copernican heliocentric view this is caused by the eccentricity of the Keplerian elliptical orbit.

The eccentric motion of the Earth has an interesting consequence. Look at Figure 1: it produced a periodic deviation of the Sun's position for a terrestrial observer compared to the case of a circular motion. The figure shows the Earth on its orbit in four positions belonging to intervals of a quarter of a year each. At the positions of perihelion $P$ and aphelion $\mathrm{A}$ there are no deviations. But at $\mathrm{B}$ and $\mathrm{C}$ a terrestrial observer perceives the Sun in a position which deviates by the angle $\Delta \phi_{\mathrm{E}}$ compared to the case if the terrestrial orbit is circular. By the daily rotation this results in a deviation in time of day . At the positions $P$ and $A$ the Sun is passing the meridian at $12 \mathrm{~h}$ noon. But at the position B the Sun has not yet reached the meridian at $12 \mathrm{~h}$; this means that the Sun is somewhat "late". Correspondingly at position $C$ the Sun will pass the meridian earlier than $12 \mathrm{~h}$. Figure 2 shows this time deviation throughout the year. Because we have good clocks now, which measure exactly the continuous flow of the time, we can measure these differences of "solar time" against the independently running time easily.

\section{Procedure for the classroom}

This variation of solar time can be checked by simple means in the classroom by observing a sun beam which passes through a little hole in a cardboard sheet which has been fixed at a window facing South. We register the time, when the centre of the Sun's image passes a line on the floor (Figure 3). This time can be read on a radio controlled clock, which is available at low cost in the shops now and which gives the time to $1 \mathrm{~s}$ exactly. By observing the Sun's image carefully when it passes the measuring line, we' may obtain this moment to about $3 \mathrm{~s}$ exactly. We need no lenses, only this piece of cardboard.

It is sufficient to repeat this measurement in the classroom once a week in a sequence of a few times, in order to obtain remarkable deviations of several minutes. This demonstrates the deviation convincingly. It shows that the Sun is not crossing the reference line at the same time each day. A longer extension of these measurements will not be 


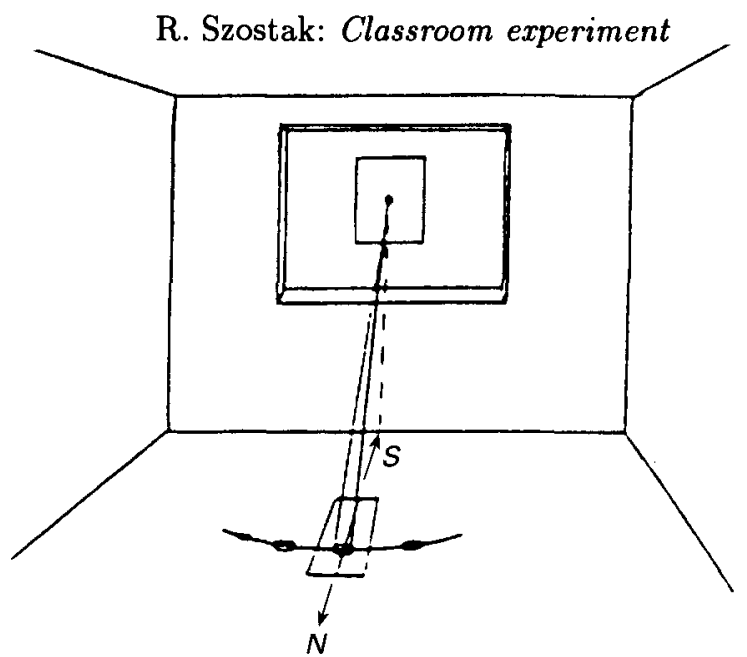

FIGURE 3.

suitable for school practice. But when the students have been convinced of the Sun's delay by their own experience, they may collect the residual data from other sources.

There is a data block cheaply available to everybody: in a normal calendar you may find the data for sunrise and sunset, at least once a week. By taking the mean value between sunrise and sunset one obtains the time, when the Sun passes the meridian. This way one finds the deviation throughout the year as shown in Figure 4, which is called the "equation of time". But unfortunately this curve is not the same as expected using Figure 2. With some imagination the expected curve can vaguely be recognized in the two big extrema at left and right. Apparently there is another effect of double periodicity superimposed as shown in Fig. 5.

In order to find out the origin of this additional effect let us consider a normal sundial. If we install a gnomon vertically on a horizontal ground, the full hours will not occur equidistantly on the clock face. An equidistant division will be obtained only, if the clock face is inclined into coincidence with the equatorial plane and if the gnomon is in coincidence with the direction of the terrestrial axis. We see, that the nonequidistant characteristic was an artefact of oblique projection, when the sundial was inclined to the Earth's axis. This deviation has evidently four zeros: at noon, at 06.00 , at 18.00 and (virtually) at midnight. So it has the expected double periodicity.

A corresponding artefact, but with regard to the period of a year, results from the inclination of the equatorial plane to the orbital plane of the Earth. For a terrestrial observer the Sun does not move during the year in the equatorial plane but in the ecliptic plane which is the plane of the Earth's orbit and is inclined by $23.5^{\circ}$ (Fig. 6). This produces the following deviation: if the Sun were continuously moving on a circle in the equatorial plane, an observer on the daily rotating Earth would see the Sun passing the meridian all the year in equidistant intervals of average solar days. But as the Sun moves along the inclined plane, it passes the meridian of the observer somewhat later on its way between $F$ and $S$ in Figure 6. Correspondingly the Sun passes the meridian somewhat earlier than the "average time", if it is on its way between $\mathbf{S}$ and $\mathbf{H}$.

This projection error $\Delta \Phi$ has four zeros during the year: the two equinoxes $F$ and $H$ for springtime and for autumn, and the solstices $S$ and $W$ for summer and winter. These are the beginnings of each of the seasons. So this function $\Delta \Phi_{P}$ possesses the double periodicity, which we looked for. From Figure 6 one can find by geometrical rules

$$
\Delta \Phi_{P}=\Phi_{\ddot{A}}-\arctan \left(\cos \epsilon \cdot \tan \Phi_{\ddot{\mathrm{A}}}\right) \text {. }
$$




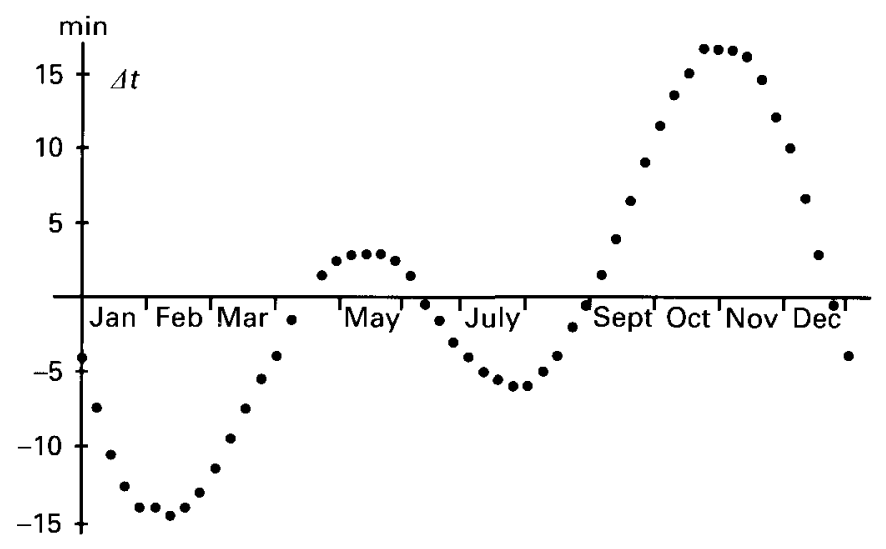

FIGURE 4.

This function can be completely calculated $\left(\epsilon=23.5^{\circ}\right)$ and converted to the time difference $\Delta t_{p}$ by the angular velocity of the terrestrial rotation, where $1^{\circ}$ corresponds to 4 min. As the zeros are well known by the dates of the equinoxes and solstices, the values of this function can be subtracted from the empirical data given in Fig. 4. By this we obtain the curve in Fig. 7, which corresponds to the expected behaviour of Fig. 2. This now contains only the influence of the eccentricity. It is the amplitude in Fig. 7 which depends directly on the eccentricity. The zeros in Figure 7 reveal immediately the dates of perihelion and of aphelion which can be identified with an error of less than 2 days. This is surprising in the case of small eccentricity.

Let us now calculate the value of the numerical eccentricity. Consider a point $C$ in Figure 8 which is reached exactly at half of the time between $A$ and $P$. Due to the second law of Kepler the areas of the elliptical parts SAC and SCP must be equal. But the calculation procedure of these areas is tedious. We exchange the shadowed parts in Figure 8 and obtain two areas of equal size of a quarter of an ellipse each. These two shadowed areas are congruent triangles except for the little curved part which in case of the small eccentricity causes an error of only $10^{-4}$. The triangle containing the angle $\Delta \Phi_{\mathrm{E}}$ offers the relation

$$
\tan \Delta \Phi_{\mathrm{E}}=\frac{2 e}{p}=\frac{2 e}{b^{2} / a}=\frac{2 e}{a}\left(\frac{a}{b}\right)^{2}=2 \epsilon \frac{a^{2}}{a^{2}-e^{2}}=\frac{2 \epsilon}{1-\epsilon^{2}} \approx 2 \epsilon
$$

where the term $\epsilon^{2} \approx 10^{-4}$ can be neglected. $\Delta \Phi_{\mathrm{E}}$ can be related to $\Delta t_{\mathrm{E}}$ by $1^{\circ}=4$ $\min$ as before. Comparison with Figure 8 shows that the date of $\mathrm{C}$ will be close to the maximum of the curve in Figure 7 . So it is justified to take the amplitude $\left(\Delta \Phi_{\mathrm{E}}\right)_{0}$ in Figure 7 for calculating

$$
\epsilon=\frac{1}{2} \tan \left(\Delta \Phi_{\mathrm{E}}\right)_{0}
$$

The amplitude in Figure 7 gives $\epsilon=0.01768$. Comparing this with the exact value $\epsilon=0.01675$ shows that we have a result which is exact to $5 \%$. Remembering that we use only dates of sunrise and sunset in a cheap calendar, this result is quite satisfying. If we take any ordinary annual data book for amateurs, we obtain results with an error of about $1 \%$.

There is still another correction to be made which is rather trivial but to be considered at the school level. The dates of sunrise and sunset given in the calendar are valid only for a certain geographical position, in our case for the town of Kassel, which has a longitude 


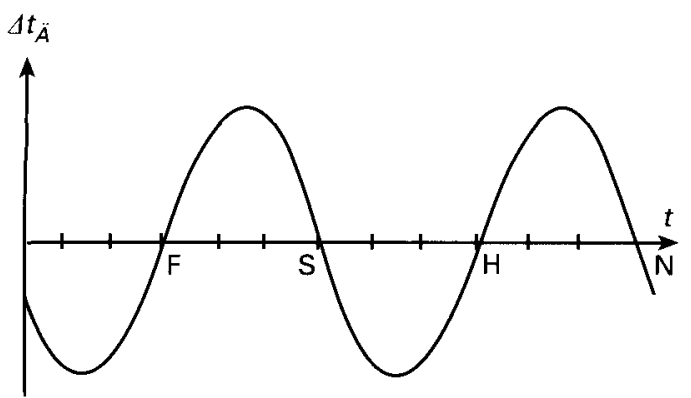

Figure 5.

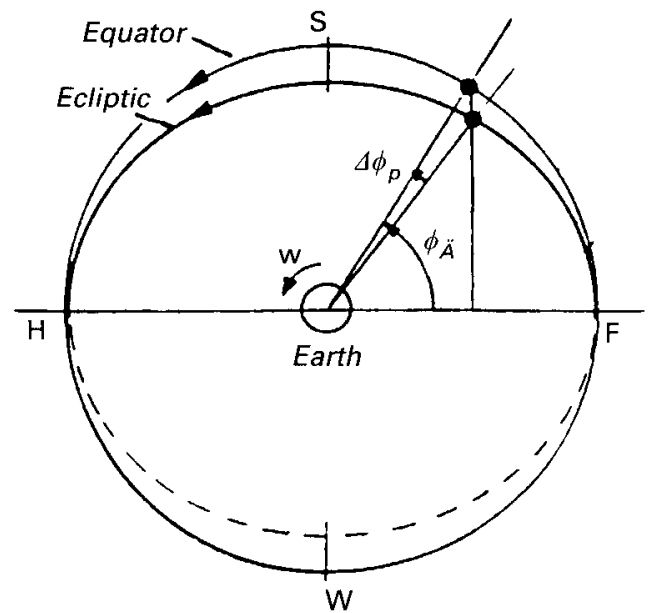

Figure 6.

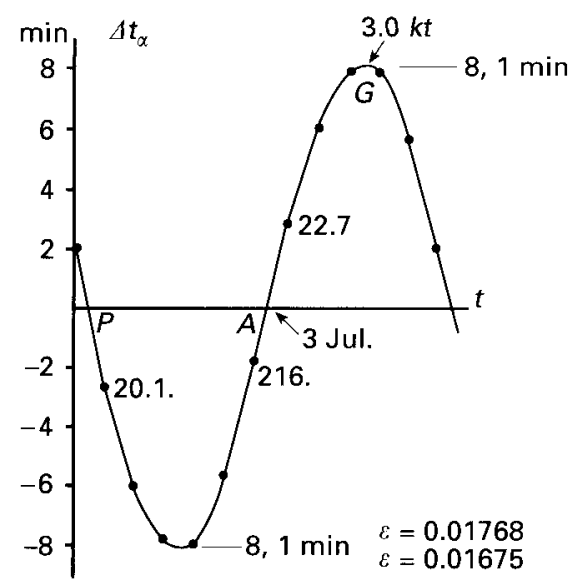

Figure 7. 


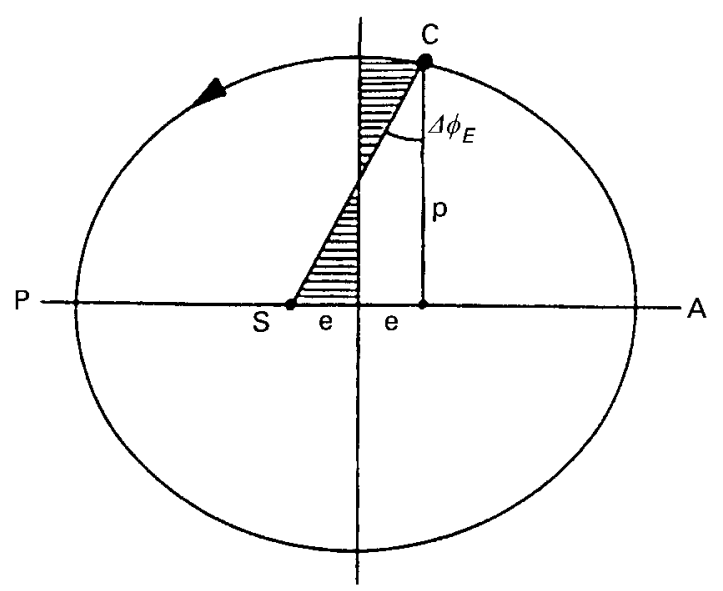

Figure 8.

of $9.5^{\circ}$ East. At this longitude the Sun passes the meridian generally somewhat later than the reference meridian for the Central European Time which is located at $15^{\circ}$ East. The difference $15^{\circ}-9.5^{\circ}$ results in a delay of $22 \mathrm{~min}$. this had been taken into account already in our data given in Figure 4 without mentioning. If this correction is not made accurately, the zero line in Figure 7 will be shifted up or down. But for calculating $\epsilon$ from this figure this shift is not important. We can omit this shift in the end because we need only the amplitude which can be read between the minimum and the maximum of Figure 7. 